\title{
A SIMPLE PARAMETRIC MODEL FOR RATING AUTOMOBILE INSURANCE OR ESTIMATING IBNR CLAIMS RESERVES
}

\author{
By Thomas Mack
}

Munich Re, Munich, FRG

\begin{abstract}
It is shown that there is a connection between rating in automobile insurance and the estimation of IBNR claims amounts because automobile insurance tariffs are mostly cross-classified by at least two variables (e.g. territory and driver class) and IBNR claims run-off triangles are always cross-classified by the two variables accident year and development year. Therefore, by translating the most well-known automobile rating methods into the claims reserving situation, some known and some unknown claims reserving methods are obtained. For instance, the automobile rating method of BAILEY and SIMON produces a new claims reserving method, whereas the model leading to the rating method called "marginal totals" produces the well-known IBNR claims estimation method called "chain ladder". A drawback of this model is the fact that it is designed for the number of claims and not for the total claims amount for which it is usually applied.

As an alternative for both, rating and claims reserving, we describe a simple but realistic parametric model for the total claims amount which is based on the Gamma distribution and has the advantage of providing the possibility of assessing the goodness-of-fit and calculating the estimation error. This method is not very well known in automobile insurance-although a satisfactory application is reported-and seems to be completely unknown in the field of claims reserving, although its execution is nearly as simple as that of the chain ladder method.
\end{abstract}

\section{KEYWORDS}

Cross-classified data; (automobile \& property) ratemaking; IBNR claims; Gamma model; maximum likelihood method.

\section{CONTENTS OF THE PAPER}

1. A short overview of some automobile rating methods

2. Some methods of estimating IBNR claims reserves and their connection to automobile rating methods 
3. A parametric model for rating automobile insurance or estimating IBNR claims reserves

4. Statistical analysis of the model

5. Improvement of the model in the case of known claims numbers

6. Final remark

App. A. Proof that the chain ladder method can be derived from the marginal totals conditions (and therefore is maximum likelihood in the Poisson case)

App. B. Estimation of the (asymptotic) error variances

Acknowledgement

References

\section{A SHORT OVERVIEW OF SOME AUTOMOBILE RATING METHODS}

In the automobile insurance tariffs of many countries several tariff variables are used, e.g. the horse-power class of the car, the bonus/malus (or no claims discount) class of the driver or the class of the territory where the car is principially garaged. In this way the portfolio of automobile insurance policies is cross-classified into a number of cells which are each supposed to be homogeneous, so that all policies of the same cell pay the same premium. For the sake of simplicity we will consider in the following only two tariff variables, which are subdivided into $m$ and $n$ classes respectively. When then have $m n$ cells labelled $(i, j), i=1, \ldots, m, j=1, \ldots, n$. Now let $n_{i j}$ be the known number of insureds (policy years) of cell $(i, j)$ and $s_{i j}$ their observed total claims amount as realization of the random variable $S_{i j}$. For some of the cells, $n_{i j}$ may be so small that it is not advisable to use $s_{i j}$ as the only basis for the calculation of the net premium $E\left(S_{i j}\right) / n_{i j}$ of that cell. Therefore one searches for marginal parameters $x_{i}, i=1, \ldots, m$, and $y_{j}, j=1, \ldots, n$, with

either $\quad x_{i} y_{j}=E\left(S_{i j}\right) / n_{i j} \quad$ (multiplicative approach),

or $\quad x_{i}+y_{j}=E\left(S_{i j}\right) / n_{i j} \quad$ (additive approach).

This also reduces the number of figures needed to describe the tariff premiums from $m n$ to $m+n$. In the following we only consider the multiplicative approach, but the methods described can easily be translated to the additive approach, too.

The problem of finding appropriate marginal parameters $x_{i}$ and $y_{j}$ is one of the classical problems of insurance mathematics. It has been known for a long time that the simple marginal averages

$$
\begin{aligned}
x_{i} & =s_{i+} / n_{i+} \\
y_{j} & =\left(s_{+j} / n_{+j}\right) /\left(s_{++} / n_{++}\right)
\end{aligned}
$$

(where a ' + ' indicates summation over the corresponding index) give a satisfactory approximation of $E\left(S_{i j}\right) / n_{i j}$ only if the tariff variables are independent. But generally this is not the case. Therefore, in the last 30 years several different methods have been proposed. We will now shortly review three of the 
most well-known mainly following the description given by VAN EEGHEN/GREUP/NIJSSEN (1983). For a more comprehensive and more recent comparative analysis see JEE (1989).

The first breakthrough was achieved by BAILEY/SIMON (1960), who estimated $x_{i}, y_{j}$ by minimizing

$$
\begin{aligned}
Q & =\sum_{i=1}^{m} \sum_{j=1}^{n}\left(s_{i j}-n_{i j} x_{i} y_{j}\right)^{2} /\left(n_{i j} x_{i} y_{j}\right) \\
& =\sum_{i=1}^{m} \sum_{j=1}^{n} n_{i j}\left(s_{i j} / n_{i j}-x_{i} y_{j}\right)^{2} /\left(x_{i} y_{j}\right),
\end{aligned}
$$

but their underlying assumption of $Q$ having (up to a factor) the distribution of a chi-square will normally not be true (see vaN EEGHEN/GREUP/NIJSSEN 1983). Moreover, it can be shown (VAN EEGHEN/NIJSSEN/RUYGT 1982) that for the minimizing parameters $x_{i}, y_{j}$ the inequalities

$$
\begin{aligned}
& \sum_{j=1}^{n} n_{i j} x_{i} y_{j} \geq \sum_{j=1}^{n} s_{i j}, \quad i=1, \ldots, m, \\
& \sum_{i=1}^{m} n_{i j} x_{i} y_{j} \geq \sum_{i=1}^{m} s_{i j}, \quad j=1, \ldots, n,
\end{aligned}
$$

hold, i.e. there results an overestimation of all marginal loss amounts (in the multiplicative case only).

Therefore BAILEY (1963) and later JUNG (1968) proposed estimating $x_{i}, y_{j}$ directly from the intuitively appealing conditions

$$
\sum_{j=1}^{n} n_{i j} x_{i} y_{j}=\sum_{j=1}^{n} s_{i j}, \quad i=1, \ldots, m,
$$

and

$$
\sum_{i=1}^{m} n_{i j} x_{i} y_{j}=\sum_{i=1}^{m} s_{i j}, \quad j=1, \ldots, n,
$$

which can be solved iteratively: starting with, for example, $y_{j}=1$, (1a) results in $x_{i}=s_{i+} / n_{i+}$, which is inserted in (1b) giving new $y_{j}$ etc. The procedure converges quickly. This method has been called "marginal totals". If the random variables $S_{i j}$ denote the number of claims instead of the total claims amount, then this method can be shown to be maximum likelihood under the assumption that all $S_{i j}$ are independent and Poisson distributed with parameter $n_{i j} x_{i} y_{j}$ (see van Eeghen/Greup/NiJssen 1983, p. 93). But for the more important case where $S_{i j}$ is the total claims amount one has no model from which the equations above derive and thus, for example, a statistical test of the goodness-of-fit cannot be designed either. 
SANT (1980) proposed estimating $x_{i}, y_{j}$ by the method of weighted least squares, i.e. by minimizing

$$
\sum_{i=1}^{m} \sum_{j=1}^{n}\left(s_{i j}-n_{i j} x_{i} y_{j}\right)^{2} / n_{i j}=\sum_{i=1}^{m} \sum_{j=1}^{n} n_{i j}\left(s_{i j} / n_{i j}-x_{i} y_{j}\right)^{2} .
$$

But the powerful tools of regression analysis like the $R^{2}$ - statistic, the analysis of residuals and the estimation of the prediction error can only be applied rigorously if all $S_{i j}$ are normally distributed with $\operatorname{Var}\left(S_{i j}\right)$ proportional to $n_{i j}$. Both assumptions are not very realistic.

Using the additive approach, the weighted least squares method leads to the same equations for the marginal parameters $x_{i}, y_{j}$ as the marginal totals method, which in this case is no longer the maximum likelihood estimator for Poisson distributed numbers of claims.

Altogether, in the case of $S_{i j}$ being claims totals all three methods described above are only of a heuristic nature without an underlying realistic model.

\section{SOME METHODS OF ESTIMATING IBNR CLAIMS RESERVES AND THEIR CONNECTION TO AUTOMOBILE RATING METHODS}

We now turn to the problem of estimating IBNR claims reserves. For an overview see VAN EEgHEN (1981) or TAYLOR (1986). Here $s_{i j}$ and $S_{i j}$ respectively-we intentionally use the same symbols as before-denote the inflation-adjusted total amount of payments made in development year $j, j=1, \ldots, n$, for accidents occurred in accident year $i, i=1, \ldots, m$. If one works with incurred amounts, $s_{i j}$ and $S_{i j}$ denote the total amount of changes in valuation made in development year $j$ on behalf of claims of accident year $i$. Working with incremental amounts we may assume that all $S_{i j}$ are independent. Typically, one has $n=m$ and $s_{i j}$ is known for all $i+j \leq m+1$ (run-off triangle), and one is interested in estimating $E\left(S_{i j}\right)$ for $i+j>m+1$. The known measure of exposure $n_{i j}$ here normally only depends on the accident year $i$, i.e. $n_{i j}=n_{i}$ (number of policies or number of claims reported in the first development year) or is even ignored (i.e. $n_{i j}=1$ for all $i, j$ ).

One of the most important ways of treating the IBNR problem is to assume a multiplicative structure of the type

$$
E\left(S_{i j}\right)=x_{i} y_{j}
$$

and to estimate the parameters $x_{i}, y_{j}$ from the triangle of known data. This way was used, for example, by DE VYLDER (1978), who estimated $x_{i}, y_{j}$ by minimizing

$$
\sum_{i, j}\left(s_{i j}-x_{i} y_{j}\right)^{2}
$$

(where the summation is for all $i, j$ where $s_{i j}$ is known). This is exactly the same method as was used by SANT (1980) in the context of automobile insurance if 
one puts all $n_{i j}=1$ there. Analogously each method which estimates the marginal parameters $x_{i}, y_{j}$ for cross-classified automobile insurance data can also be translated into a method for estimating the IBNR claims reserve. One only must take the different pattern of known data (triangle instead of rectangle) into account.

Let us consider as further example the method of marginal totals.

Again working with $n=m$ and $n_{i j}=1$, we get the conditions

$$
\begin{array}{ll}
\text { (H) } \quad \sum_{j} x_{i} y_{j}=\sum_{j} s_{i j}, & i=1, \ldots, m, \\
\left(V_{j}\right) \quad \sum_{i} x_{i} y_{j}=\sum_{i} s_{i j}, & j=1, \ldots, m,
\end{array}
$$

where the summation is for those indices where the corresponding $s_{i j}$ are known (i.e. in the case of a full triangle $j$ runs from 1 to $m+1-i$ and $i$ from 1 to $m+1-j$ ). The same equations are also obtained if one derives the maximum likelihood equations in the Poisson case.

Because of the triangular structure, the above equations can here be solved recursively: We start with the general observation that the solution of this type of problem is only unique up to a multiplicative constant $c \# 0$ because if $x_{i}, y_{j}$ is a solution, $x_{i} c, y_{j} / c$ is a solution as well. Therefore, without loss of generality we can put $y_{1}+\ldots+y_{m}=1$. Then using equation $\left(H_{1}\right)$ we have $x_{1}=s_{1+}$. From equation $\left(V_{m}\right)$ we get $y_{m}=s_{1 m} / x_{1}$. Then $\left(H_{2}\right)$ yields $x_{2},\left(V_{m-1}\right)$ yields $y_{m-1}$ etc.

But it is also possible to derive a direct formula for the unknown mean claims amount $E\left(S_{i j}\right)=x_{i} y_{j}$. For $h>m+1-i$ it can be shown (see KREMER 1985 , p. 133-136, or Appendix A where a shorter proof is given) that

$$
x_{i} y_{h}=\left(\sum_{j=1}^{m+1-i} s_{i j}\right) \cdot f_{m+2-i} \cdot f_{m+3-i} \cdots f_{h-1} \cdot\left(f_{h}-1\right)
$$

where $\quad f_{j}=\left(\sum_{k=1}^{m+1-j} \sum_{l=1}^{j} s_{k l}\right) /\left(\sum_{k=1}^{m+1-j} \sum_{l=1}^{j-1} s_{k l}\right), \quad j=2, \ldots, m$.

If one realizes that $\sum_{l=1}^{j} s_{k l}$ is the accumulated claims amount of accident year $k$ known at the end of development year $j$, one sees that we have just obtained the well-known chain ladder method which is thus shown to be the same as the marginal totals method for $n_{i j}=1$. Furthermore, from the marginal totals conditions $\left(H_{i}\right),\left(V_{j}\right)$ one easily sees that an incorporation (analogously to (la) and (lb)) of the known exposure $n_{i}$ into the estimation of the IBNR claims reserve can be dispensed with, as $n_{i}$ can be amalgamated with the marginal parameter $x_{i}$ (in the multiplicative approach only), whereas the 
application of the chain ladder method to the claims ratios $s_{i j} / n_{i}$ assumes a different model.

It is interesting to note that the analogue of the BAILEY-SIMON method seems to have never been published as a method for estimating the IBNR claims reserve.

Another interesting point is the fact that in the context of IBNR claims estimation only the multiplicative approach seems to have been used, although several applications to automobile rating indicate that there the additive approach might give a better fit (see e.g. CHANG/FAIRLEY 1979). A special feature of the additive approach is that it may lead to negative values $E\left(S_{i j}\right)=x_{i}+y_{j}$. This would make no sense in the ratemaking situation but in the case of claims reserving it can be very realistic (settlement gains).

Clearly, also in the context of claims reserving the least squares method and the marginal totals method (and, of course, the BAILEY-SIMON method) could be carried through with the additive approach, too, both producing an identical set of equations for $x_{i}, y_{j}$ as has already been mentioned in the section on automobile rating.

There is a natural connection between the multiplicative and the additive approaches because, through the log-transformation,

becomes

$$
s_{i j} / n_{i j} \approx x_{i} y_{j}
$$

$$
\log \left(s_{i j} / n_{i j}\right) \approx \log \left(x_{i}\right)+\log \left(y_{j}\right) .
$$

This means that an estimate for $E\left(S_{i j} / n_{i j}\right)$ can be established by applying an additive approach to the log-transformed data $\log \left(s_{i j} / n_{i j}\right)$ and by transforming back the obtained solution $\log \left(x_{i}\right), \log \left(y_{j}\right)$ using the exponential function. This was done by CHANG/FAIRLEY (1979) for automobile rating and by KREMER (1982) (see also ZEHNwIRTH 1989) for claims reserving (with $n_{i j}=1$ ). For the solution of the transformed (additive) problem, both used the method of (weighted) least squares (here giving the same result as the marginal totals method) in order to estimate the marginal parameters $\log \left(x_{i}\right), \log \left(y_{j}\right)$.

As ZEHNWIRTH (1989) points out, this procedure contains an implicit distributional assumption: In order to fulfill the conditions of normality and homoscedasticity for the least squares estimation of the parameters $\log \left(x_{i}\right)$ and $\log \left(y_{j}\right)$, it has to be assumed that $\log \left(S_{i j} / n_{i j}\right)$ has a normal distribution with mean value $\log \left(x_{i}\right)+\log \left(y_{j}\right)$ and a variance which is proportional to $1 / n_{i j}$. This implies that $S_{i j} / n_{i j}$ is assumed to have a lognormal distribution. CHANG/FaIRLEY and KREMER did not take this implicit distributional assumption into account. Therefore, they systematically underestimated $E\left(S_{i j} / n_{i j}\right)$ as they used $x_{i} y_{j}=\exp \left(\log \left(x_{i}\right)+\log \left(y_{j}\right)\right)$, which is the median of the lognormal distribution whereas the expected value is $x_{i} y_{i} \exp \left(\sigma_{i j}^{2} / 2\right)$ with $\sigma_{i j}^{2}=\operatorname{Var}\left(\log \left(S_{i j} / n_{i j}\right)\right)$. As stated above, we have homoscedasticity if we assume that $\sigma_{i j}^{2}=\sigma^{2} / n_{i j}$, where $\sigma^{2}$ can be estimated by

$$
\sum_{i, j} n_{i j}\left(\log \left(s_{i j} / n_{i j}\right)-\log \left(x_{i} y_{j}\right)\right)^{2} /(c-m-n+1),
$$


which is just the expression to be minimized by the least squares method. Here $c$ denotes the number of cells where $s_{i j}$ is known.

Unfortunately, we have lost the multiplicative structure, as generally $E\left(S_{i j} / n_{i j}\right)=x_{i} y_{j} \exp \left(\sigma_{i j}^{2} / 2\right)$ cannot be cast into the form $E\left(S_{i j} / n_{i j}\right)=\tilde{x}_{i} \tilde{y}_{j}$ anymore.

Whereas all the models discussed before have been shown to be only of a heuristic nature both in automobile rating and in claims reserving, the lognormal model relies on a parametric assumption for $S_{i j}$, and the instruments of regression analysis can be used to check this assumption against the data. In the next section another method is given which relies on a reasonable distributional model and therefore also allows the application of various important and useful statistical tools. This model has two advantages over the lognormal model. First, it is not just any model for $S_{i j}$ but can be traced back to a micro-model for the total claims amount of each single insured unit and can therefore be expected to be realistic. Second, we can choose either the multiplicative or the additive structure for $E\left(S_{i j} / n_{i j}\right)$, whereas the lognormal model yields neither of these structures.

\section{A PARAMETRIC MODEL FOR RATING AUTOMOBILE INSURANCE OR ESTIMATING IBNR CLAIMS RESERVES}

We use the same notations as before, i.e. we have $m n$ cells labelled $(i, j)$, each with known measure of exposure $n_{i j}$ (possibly independent of $j$ in the case of claims reserving) and with total claims amount variable $S_{i j}$ (realization $s_{i j}$ ). In the case of claims reserving we know the realizations $s_{i j}$ in the run-off triangle only. We now assume, following TER BERG (1980), that the total claims amount $R_{i j k}$ of each unit $k=1, \ldots, n_{i j}$ of cell $(i, j)$ has a Gamma distribution with mean value $m_{i j}$ (independent of $k$ ) and shape parameter $\alpha$ (independent of $i, j, k)$, i.e. with probability density function

$$
f_{i j}(z)=\exp \left(-\alpha z / m_{i j}\right) z^{\alpha-1}\left(\alpha / m_{i j}\right)^{\alpha} / \Gamma(\alpha)
$$

(here the usual representation of the Gamma density has been reparametrized in order to implement the mean value $m_{i j}$ directly as a parameter). Because in practice many units $k$ will have a realization $r_{i j k}=0$ of $R_{i j k}$, the shape parameter $\alpha$ has to be conceived of as smaller than 1 in order to attribute a high probability to the neighbourhood of $z=0$ (for instance, we have prob $\left(R_{i j k} \leq m_{i j} / 10\right)=0.79$ for $\left.\alpha=0.05\right)$. Assuming that all $n_{i j}$ units of cell $(i, j)$ are independent, our distributional assumption implies that $S_{i j}=R_{i j 1}+R_{i j 2}+\ldots$ also has a Gamma distribution but with mean value $n_{i j} m_{i j}$ and shape parameter $n_{i j} \alpha$. And this is the distribution we shall work with in the following, because we usually know only the realizations $s_{i j}$ of $S_{i j}$ and not those of $R_{i j k}$. The assumption that the shape parameter $\alpha$ is the same for the units of all cells may seem questionable in some cases. But this should be detected by testing the goodness-of-fit (see next Section). 
In the multiplicative approach we assume furthermore that $m_{i j}$ can be displayed in the form $m_{i j}=x_{i} y_{j}$ with unknown parameters $x_{i}, y_{j}$, which we shall estimate with the maximum likelihood method.

Assuming that all $S_{i j}$ are independent, the likelihood function on the basis of the realizations $s_{i j}>0$ is given by

$$
L=\prod_{i, j} \exp \left(-\frac{\alpha s_{i j}}{x_{i} y_{j}}\right)\left(\frac{\alpha s_{i j}}{x_{i} y_{j}}\right)^{n_{i j} \alpha} /\left(s_{i j} \Gamma\left(n_{i j} \alpha\right)\right) .
$$

Therefore the loglikelihood function is

$\log (L)=\sum\left\{-\alpha s_{i j} /\left(x_{i} y_{j}\right)+n_{i j} \alpha \log \left(\alpha s_{i j}\right)-n_{i j} \alpha \log \left(x_{i} y_{j}\right)-\log \left(s_{i j} \Gamma\left(n_{i j} \alpha\right)\right)\right\}$

(where the summation is for all $i, j$ where $s_{i j}$ is known). The maximum likelihood estimator are those values $x_{i}, y_{j}, \alpha$ which maximize $L$ or equivalently $\log (L)$. They are given by the equations

$$
\begin{array}{ll}
0=\partial \log (L) / \partial x_{i}=\alpha \sum_{j}\left(s_{i j} /\left(x_{i}^{2} y_{j}\right)-n_{i j} / x_{i}\right), & i=1, \ldots, m, \\
0=\partial \log (L) / \partial y_{j}=\alpha \sum_{i}\left(s_{i j} /\left(x_{i} y_{j}^{2}\right)-n_{i j} / y_{j}\right), & j=1, \ldots, n,
\end{array}
$$

which show that the last condition $\partial \log (L) / \partial \alpha=0$ is not needed for the calculation of the likelihood estimator for $x_{i}, y_{j}$, which can immediately be seen to be given by

$$
\begin{cases}x_{i}=\frac{1}{n_{i+}} \sum_{j} \frac{s_{i j}}{y_{j}}, & i=1, \ldots, m, \\ y_{j}=\frac{1}{n_{+j}} \sum_{i} \frac{s_{i j}}{x_{i}}, & j=1, \ldots, n .\end{cases}
$$

These equations have a high intuitive appeal. For, considering the goal of approximating $s_{i j}$ by $n_{i j} x_{i} y_{j}$, we see that this amounts to approximating $s_{i j} /\left(n_{i j} y_{j}\right)$ by $x_{i}$ and therefore the $n_{i j}$-weighted mean of $s_{i j} /\left(n_{i j} y_{j}\right), j=1, \ldots, n$, should be a reasonable estimator for $x_{i}$.

Also, equations (2) are not new. They have already been used by vaN EEGHEN/Nussen/RUYGT (1982). They call them the "direct method" and write (on page 111):

"This set of equations are a direct translation of the intuitive calculations presented ... by F. K. GREGORIUS. In fact, a solution is found when iteratively calculating the values $x_{i}$ and $y_{j}$ by means of the formulae given in (2) by letting $y_{j}=1(j=1, \ldots, n)$ be the starting value. The procedure converges rapidly. 
We may rewrite (2) as

$$
\begin{aligned}
& \sum_{j} n_{i j} x_{i}=\sum_{j} s_{i j} / y_{j}, \quad i=1, \ldots, m, \\
& \sum_{i} n_{i j} y_{j}=\sum_{i} s_{i j} / x_{i}, \quad j=1, \ldots, n,
\end{aligned}
$$

which is similar but not equivalent to (la) and (1b).

As yet, we have not been able to find an argument why a 'satisfactory' solution should (approximately) satisfy (2) ...

The method was more or less developed as a first try and we were surprised to see, that, once formalized, it produced practically the same results as the method of marginal totals."

So much for the quotation from van EEghen/NiJsSEN/RUYGT (1982).

One year later the Dutch actuaries found an argument for their method because the booklet of VAN EEGHEN, Greup and NIJSSEN (1983) contains on page 109 a small hint saying that the assumption of a Gamma distribution for $R_{i j k}$ would lead to the "direct method". But there, as in TER BERG (1980), a much more general regression model is considered, of which our simple cross-classified situation is just a special case. Moreover, these authors have concentrated on ratemaking, whereas we want to emphasize the applicability to claims reserving, too.

Finally, it is interesting to note that the likelihoood equations for the additive approach

$$
\begin{aligned}
& \sum_{j}\left(s_{i j} /\left(x_{i}+y_{j}\right)^{2}-n_{i j} /\left(x_{i}+y_{j}\right)\right)=0, \quad i=1, \ldots, m \\
& \sum_{i}\left(s_{i j} /\left(x_{i}+y_{j}\right)^{2}-n_{i j} /\left(x_{i}+y_{j}\right)\right)=0, \quad j=1, \ldots, n,
\end{aligned}
$$

must be solved with the help of, for example, the NEWTON-RAPHSON numerical method. Moreover, these equations are different from those suggested by the "direct method":

$$
\begin{aligned}
& x_{i}=\sum_{j}\left(s_{i j}-n_{i j} y_{j}\right) / n_{i+}, \quad i=1, \ldots, m, \\
& y_{j}=\sum_{i}\left(s_{i j}-n_{i j} x_{i}\right) / n_{+j}, \quad j=1, \ldots, n .
\end{aligned}
$$

\section{STATISTICAL ANALYSIS OF THE MODEL}

This parametric approach with a realistic distributional assumption enables us to use many tools for the statistical analysis, as has been clearly set out by 
Albrecht (1983), who describes the case $\alpha=1$ in considerable detail but again as a general regression model. Besides the consistent and (asymptotically) efficient estimation of the model parameters, we have the possibility of testing the significance of the tariff variables with the likelihood ratio test (see AlBRECHT (1983) for details), we can calculate the error variances of the parameter estimators and we can check the goodness-of-fit. We first consider the goodness-of-fit: According to our model, $S_{i j}$ has a Gamma distribution with $E\left(S_{i j}\right)=n_{i j} m_{i j}$ and $\operatorname{Var}\left(S_{i j}\right)=n_{i j} m_{i j}^{2} / \alpha$. The higher the shape parameter $n_{i j} \alpha$ of this distribution, the closer it is to the normal distribution. If all $S_{i j}$ are approximately normally distributed the statistic

$$
\begin{aligned}
\sum_{i, j} \frac{\left(S_{i j}-E\left(S_{i j}\right)\right)^{2}}{\operatorname{Var}\left(S_{i j}\right)} & =\alpha \sum_{i, j} \frac{\left(S_{i j} /\left(x_{i} y_{j}\right)-n_{i j}\right)^{2}}{n_{i j}} \\
& =\alpha \sum_{i, j} n_{i j}\left(\frac{S_{i j}}{n_{i j} x_{i} y_{j}}-1\right)^{2}
\end{aligned}
$$

is, under the hypothesis of our model, approximately at chi-square with $c-m-n$ degrees of freedom, where $c$ is the number of cells where $s_{i j}$ is known.

The special form of this statistic allows its application without having estimated $\alpha$. For this purpose we fix $\alpha$ in such a way that the value of the statistic is just below the (say) 0.95 -fractile of the chi-square distribution. If using this value of $\alpha$ a normality condition like " $n_{i j} \alpha>10$ " is fulfilled for nearly all cells, we may be satisfied with the goodness-of-fit of the model. But we have to realize that this goodness-of-fit test only checks the fit of aggregated figures and cannot test the distributional assumptions within the cells.

Applying this procedure to SANT'S (1980) collision data (126 cells) we get $\alpha(<)=0.021$ and the three lowest values of $n_{i j} \alpha$ turn out to be 6.8, 9.4 and 11.5 , so we may accept the multiplicative Gamma model. Using CHANG/FaIRLEY's (1979) combined compulsory data (105 cells), we get $\alpha(<)=0.0094$ and have 9 cells were the resulting value of $n_{i j} \alpha$ is lower than 10 , the lowest being 4.5 , so the fit is less satisfactory.

A simple formula for an estimator of $\alpha$ is given by the method of moments, i.e. by equating the variances

$$
\sum_{i, j}\left(s_{i j}-n_{i j} x_{i} y_{j}\right)^{2}=\sum_{i, j} n_{i j}\left(x_{i} y_{j}\right)^{2} / \alpha .
$$

This yields $\alpha=0.014$ for Sant's data and $\alpha=0.0093$ for Chang/Fairley's data.

Strictly speaking we should use the likelihood estimator for $\alpha$. We then must solve the likelihood equation

$$
0=\partial \log (L) / \partial \alpha=\sum_{i, j} n_{i j}\left\{\log \left(\alpha s_{i j}\right)-\log \left(x_{i} y_{j}\right)-\psi\left(n_{i j} \alpha\right)\right\} .
$$


Here equations (2) have been used to obtain $\Sigma n_{i j}=\Sigma s_{i j} /\left(x_{i} y_{j}\right) . \psi(z)=$ $\Gamma^{\prime}(z) / \Gamma(z)$ denotes the digamma function, for which the asymptotic approximation

$$
\psi(z) \approx \log (z)-(2 z)^{-1}-z^{-2} / 12
$$

exists which even for arguments as low as $z \geq 4$ is exact to 4 decimal places. This approximation yields as the solution of the likelihood equation

with

$$
\alpha \approx\left(c+\sqrt{c^{2}+a b}\right) / a
$$

$$
\begin{aligned}
& a=4 \sum_{i, j} n_{i j} \log \left(n_{i j} x_{i} y_{j} / s_{i j}\right)>0, \\
& b=\sum_{i, j}\left(3 n_{i j}\right)^{-1} \\
& c=\sum_{i, j} 1=\text { number of cells where } s_{i j} \text { is known. }
\end{aligned}
$$

Applied to Sant's data this yields $\alpha \approx 0.0202$. For Chang/Fairley's data we get $\alpha \approx 0.0097$. If we have some small exposures $n_{i j}$ such that $n_{i j} \alpha<4$, we should refine the approximation of the digamma function by using the recursion $\psi(z)=\psi(z+1)-1 / z$ and by including more terms of the approximation series. Then a direct formula for $\alpha$ cannot be given anymore. We must therefore solve the likelihood equation iteratively with the NEWTON-RAPHSON method.

Having estimated $\alpha$, we are also in the position to calculate the estimation error of the estimators for $x_{i}$ and $y_{j}$. This is done in Appendix B.

According to the experience of the Dutch actuaries, the results of applying the "direct method" to automobile insurance data are rather close to the results obtained by the marginal totals method. Translated to the IBNR claims reserving problem this means that the "direct method" results will be similar to the chain ladder results. But with the "direct method" we can additionally make use of the aforementioned advantages. Moreover, the formulae provide the possibility of taking the exposure $n_{i}$ of accident year $i$ into account (which is different from the situation with the chain ladder). And perhaps the goodness-of-fit statistic or the size of the likelihood function gives an indication to answering the question "additive or multiplicative?" Because of these advantages of the parametric method we believe that before using a rather heuristic method like BAILEY/SIMON or chain ladder one should examine whether the parametric method fits the data.

\section{IMPROVEMENT OF THE MODEL IN THE CASE OF KNOWN CLAIMS NUMBERS}

Especially in the claims reserving situation we will often have difficulties in finding an adequate measure $n_{i j}$ of exposure. 
Therefore mostly $n_{i j}=n_{i}$ or even $n_{i j}=1$ is taken. However, this is not satisfactory because the exposure to further payments or changes in valuation varies in fact rather strongly over the development years. Therefore, a more meaningful measure of exposure will be the number $t_{i j}$ of those claims of accident year $i$ where there is a change in amount during development year $j$. These data $t_{i j}, i+j \leq m+1$, are often available in practice.

Rating in property insurance presents a similar problem. There, even the risks of the same cell vary greatly with respect to their size, which is usually measured by the sum insured. Therefore, the number of risks is not a good measure for the exposure of a cell $(i, j)$, and the sum insured is taken instead. But then an assumption of our micro-model is not fulfilled anymore because the "units" of sum insured are not independent, as a single risk consists of several such units. We therefore must abandon our micro-model and try directly whether the Gamma model for $S_{i j}$ with mean value $E\left(S_{i j}\right)=n_{i j} x_{i} y_{j}$ and shape parameter $n_{i j} \alpha$ fits the data if $n_{i j}$ is the sum insured. The parameter $\alpha$ then does not have a specific interpretation anymore. But if we know additionally the total number $t_{i j}$ of claims of cell $(i, j)$ we can apply the following stepwise approach which assumes a Gamma distribution (with shape parameter $\alpha$ ) not for the total claims amount per risk unit but for the amount of each single claim. Of course, this procedure can also be applied in automobile ratemaking if the number $t_{i j}$ of claims is available.

In these situations we should use $t_{i j}$-the corresponding random variable is denoted by $T_{i j}$-as an additional measure of exposure and adopt the following three-steps-approach, which follows the ideas of AlBRECHT (1983): In the first step we take the observed number $t_{i j}$ of claims of cell $(i, j)$ as the measure of exposure and assume that the size of each corresponding amount has a Gamma distribution with mean value $m_{i j}=x_{i} y_{j}$ and shape parameter $\alpha$. Then we are in our original model (with $n_{i j}$ replaced with $t_{i j}$ ) leading to the direct method. This yields smoothed average claims amounts $x_{i} y_{j}$. In the second step we smooth the $t_{i j}$ by assuming that all $T_{i j}$ are independent of each other and that each $T_{i j}$ has a Poisson distribution with parameter $n_{i j} v_{i} w_{j}$ (here using the 'old' measure of exposure). Then the maximum likelihood estimator of $v_{i}, w_{j}$ on basis of the realizations $t_{i j}$ is given by the equations (1a) and (1b) with $x_{i}, y_{j}, s_{i j}$ replaced with $v_{i}, w_{j}, t_{i j}$ respectively. This yields smoothed numbers $n_{i j} v_{i} w_{j}$ of claims. In the last step, $E\left(S_{i j}\right)$ is estimated by $n_{i j} v_{i} w_{j} x_{i} y_{j}$ implying that in each cell the number of claims is independent of the average claims amount.

\section{FINAL REMARK}

In the context of this paper we should point out the following further connection between rating methods and claims reserving methods. Another important rating method which smoothes the claims experience of several tariff classes is the Bühlmann-Straub credibility model. It also uses a cross-classifying approach by the two dimensions 'tariff classes' and 'observation years'. 
Therefore, one will presume that it could also be translated into a method for estimating IBNR claims reserves. But there is a difficulty because the Bühlmann-Straub model assumes that the average claims amount $S_{i j} / n_{i j}$ of tariff class $i$ has the same expected value over all years $j$, whereas in the run-off triangle the expected value of the average claims amount $S_{i j} / n_{i}$ of accident year $i$ and development year $j$ varies in a certain but unknown pattern over the development years. However, this difficulty can be overcome in such a way that the Bühlmann-Straub model can directly be used for claims reserving, too (see MACK 1990).

\section{APPENDIX A}

PROOF THAT THE CHAIN LADDER METHOD CAN BE DERIVED FROM THE MARGINAL TOTALS CONDITIONS (AND THEREFORE IS MAXIMUM LIKELIHOOD IN THE POISSON CASE)

We show that the chain ladder method

$$
x_{i} y_{h}=\left(\sum_{j=1}^{m+1-i} s_{i j}\right) \cdot f_{m+2-i} \cdot f_{m+3-i} \cdots f_{h-1} \cdot\left(f_{h}-1\right), \quad h>m+i-1,
$$

with

$$
f_{j}=\left(\sum_{k=1}^{m+1-j} \sum_{l=1}^{j} s_{k l}\right) /\left(\sum_{k=1}^{m+1-j} \sum_{l=1}^{j-1} s_{k l}\right), \quad j=2, \ldots, m,
$$

can be deduced from the marginal conditions

$$
\begin{aligned}
& \sum_{j=1}^{m+1-i} x_{i} y_{j}=\sum_{j=1}^{m+1-i} s_{i j}, \quad i=1, \ldots, m, \\
& \sum_{i=1}^{m+1-j} x_{i} y_{j}=\sum_{i=1}^{m+1-j} s_{i j}, \quad j=1, \ldots, m .
\end{aligned}
$$

Let $c_{i j}=\sum_{l=1}^{j} x_{i} y_{l}$ and $b_{i j}=\sum_{l=1}^{j} s_{i l}(i+j \leq m+1)$ denote the expected and the observed accumulated claims amount of accident year $i$ at the end of development year $j$ respectively. Then conditions $\left(H_{i}\right)$ can be written shortly as $c_{i, m+1-i}=b_{i, m+1-i}$. For $h>m+1-i$ we have

$$
c_{i h}=c_{i, m+1-i} \cdot \frac{c_{i, m+2-i}}{c_{i, m+1-i}} \cdots \frac{c_{i h}}{c_{i, h-1}} .
$$

Therefore

$$
x_{i} y_{h}=c_{i h}-c_{i, h-1}
$$




$$
\begin{aligned}
& =\mathrm{c}_{\mathrm{i}, m+1-i} \cdot \frac{c_{i, m+2-i}}{c_{i, m+1-i}} \cdots \frac{c_{i, h-1}}{c_{i, h-2}} \cdot\left(\frac{c_{i h}}{c_{i, h-1}}-1\right) \\
& =\left(\sum_{j=1}^{m+1-i} s_{i j}\right) \cdot \frac{c_{i, m+2-i}}{c_{i, m+1-i}} \cdots \frac{c_{i, h-1}}{c_{i, h-2}} \cdot\left(\frac{c_{i h}}{c_{i, h-1}}-1\right)
\end{aligned}
$$

and we have only to show that $c_{i j} / c_{i, j-1}=f_{j}$. Because of

$$
\frac{c_{i j}}{c_{i, j-1}}=\frac{\sum_{l=1}^{j} y_{l}}{\sum_{l=1}^{j-1} y_{l}}=\frac{\left(\sum_{k=1}^{m+1-j} x_{k}\right)\left(\sum_{l=1}^{j} y_{l}\right)}{\left(\sum_{k=1}^{m+1-j} x_{k}\right)\left(\sum_{l=1}^{j-1} y_{l}\right)}=\frac{\sum_{k=1}^{m+1-j} c_{k j}}{\sum_{k=1}^{m+1-j} c_{k, j-1}}
$$

and of

$$
f_{j}=\left(\sum_{k=1}^{m+1} b_{k j}\right) /\left(\sum_{k=1}^{m+1-j} b_{k, j-1}\right)
$$

it is enough to show that

$$
\sum_{k=1}^{m+1-j} c_{k j}=\sum_{k=1}^{m+1-j} b_{k j}
$$

and

$$
\sum_{k=1}^{m+1-j} c_{k, j-1}=\sum_{k=1}^{m+1-j} b_{k, j-1}
$$

hold for $j=2, \ldots, m$. We show this by recursion from $j=m$ to $j=2$ : $\left(A_{m}\right)$, i.e. $c_{1 m}=b_{1 m}$, holds because of $\left(H_{1}\right)$.

$\left(B_{j}\right)$ follows from $\left(A_{j}\right)$ and $\left(V_{j}\right)$ as

$$
\begin{aligned}
\sum_{k=1}^{m+1-j} c_{k, j-1} & =\sum_{k=1}^{m+1-j}\left(c_{k j}-x_{k} y_{j}\right)=\sum_{k=1}^{m+1-j} c_{k j}-\sum_{k=1}^{m+1-j} x_{k} y_{j} \\
& =\sum_{k=1}^{m+1-j} b_{k j}-\sum_{k=1}^{m+1-j} s_{k j}=\sum_{k=1}^{m+1-j}\left(b_{k j}-s_{k j}\right)=\sum_{k=1}^{m+1-j} b_{k, j-1} .
\end{aligned}
$$

Finally, $\left(A_{j-1}\right)$ follows from $\left(B_{j}\right)$ and $\left(H_{m+2-j}\right)$ as

$$
\begin{aligned}
\sum_{k=1}^{m+2-j} c_{k, j-1} & =\sum_{k=1}^{m+1-j} c_{k, j-1}+c_{m+2-j, j-1} \\
& =\sum_{k=1}^{m+1-j} b_{k, j-1}+b_{m+2-j, j-1}=\sum_{k=1}^{m+2-j} b_{k, j-1} .
\end{aligned}
$$

This completes the proof. 


\section{APPENDIX B \\ ESTIMATION OF THE (ASYMPTOTIC) ERROR VARIANCES}

We have estimated the marginal parameters $x_{i}, y_{j}$ with

either $\quad x_{i} y_{j}=E\left(S_{i j} / n_{i j}\right) \quad$ (multiplicative approach)

or $\quad x_{i}+y_{j}=E\left(S_{i j} / n_{i j}\right) \quad$ (additive approach).

by the maximum likelihood method and now want to know how precise these estimates are, i.e. we want to calculate $\operatorname{Var}\left(X_{i}\right), \operatorname{Var}\left(Y_{j}\right), \operatorname{Var}\left(X_{i} Y_{j}\right)$ or $\operatorname{Var}\left(X_{i}+Y_{j}\right)$ where $X_{i}$ and $Y_{j}$ denote the random variables corresponding to the estimators for $x_{i}$ and $y_{j}$ respectively. A standard result of maximum likelihood theory states that under certain regularity conditions which are fulfilled here, the following holds true: If a parameter vector $\Theta=\left(\Theta_{1}, \ldots, \Theta_{r}\right)$ is estimated by the maximum likelihood method, the obtained estimator $\hat{\Theta}$ has asymptotically a normal distribution with mean value $\Theta$ and with a covariance matrix which is equal to the inverse of the information matrix

$$
I(\Theta)=E\left(-\frac{\partial^{2} \log (L)}{\partial \Theta_{i} \partial \Theta_{j}}\right)_{i, j}
$$

where $L=L(\Theta)$ is the likelihood function.

In our case we have $\Theta=\left(x_{2}, \ldots, x_{m}, y_{1}, \ldots, y_{n}\right)$ where we have omitted $x_{1}$ without loss of generality in order to obtain a unique solution of the likelihood equations and have considered $\alpha$ as being known. (For the case of $\alpha$ being included in $\Theta$, TER BERG (1980) has shown that this does not change the calculation of $\operatorname{Var}\left(X_{i}\right)$, $\operatorname{Var}\left(Y_{j}\right)$ and $\left.\operatorname{Cov}\left(X_{i}, Y_{j}\right)\right)$. We now have

$$
\begin{aligned}
& \operatorname{Cov}\left(X_{2}, \ldots, X_{m}, Y_{1}, \ldots, Y_{n}\right) \approx I\left(x_{2}, \ldots, x_{m}, y_{1}, \ldots, y_{n}\right)^{-1}=: I^{-1} \\
& \approx I\left(\hat{x}_{2}, \ldots, \hat{x}_{m}, \hat{y}_{1}, \ldots, \hat{y}_{n}\right)^{-1}=: \hat{I}^{-1}
\end{aligned}
$$

where $\hat{x}_{2}, \ldots, \hat{y}_{n}$ denote the estimated values of the true parameters $x_{2}, \ldots, y_{n}$. From $\hat{I}^{-1}$ we directly obtain asymptotic approximative values for $\operatorname{Var}\left(X_{i}\right)$, $\operatorname{Var}\left(Y_{j}\right)$ and $\operatorname{Cov}\left(X_{i}, Y_{j}\right)$. This also gives immediately an approximation for

$$
\operatorname{Var}\left(X_{i}+Y_{j}\right)=\operatorname{Var}\left(X_{i}\right)+2 \operatorname{Cov}\left(X_{i}, Y_{j}\right)+\operatorname{Var}\left(Y_{j}\right)
$$

which we want to know in the additive approach. In order to obtain $\operatorname{Var}\left(X_{i} Y_{j}\right)$ for the multiplicative approach, we make use of a general theorem on the higher moments of normally distributed variables (see e.g. RICHTER 1966, p. 369) to get

$$
\begin{aligned}
\operatorname{Var}\left(X_{i} Y_{j}\right) \approx & \operatorname{Var}\left(X_{i}\right) \operatorname{Var}\left(Y_{j}\right)+\left(\operatorname{Cov}\left(X_{i}, Y_{j}\right)\right)^{2}+\operatorname{Var}\left(X_{i}\right)\left(E\left(Y_{j}\right)\right)^{2}+ \\
& +2 E\left(X_{i}\right) \operatorname{Cov}\left(X_{i}, Y_{j}\right) E\left(Y_{j}\right)+\left(E\left(X_{i}\right)\right)^{2} \operatorname{Var}\left(Y_{j}\right)
\end{aligned}
$$

(which holds exactly if $X_{i}$ and $Y_{j}$ are normally distributed). This can be calculated from $\hat{I}^{-1}$ and from $E\left(X_{i}\right) \approx \hat{x}_{i}, E\left(Y_{j}\right) \approx \hat{y}_{j}$. 
Therefore, the only thing left to do is the calculation of $I$ and $I^{-1}$ Concentrating again on the multiplicative approach, the loglikelihood function is

$$
\log (L)=-\sum_{i, j}\left(\alpha S_{i j} /\left(x_{i} y_{j}\right)+\alpha n_{i j} \log \left(x_{i} y_{j}\right)+g\left(\alpha, n_{i j}, S_{i j}\right)\right)
$$

and yields (using $E\left(S_{i j}\right)=n_{i j} x_{i} y_{j}$ and the Kronecker symbol $\delta_{i j}$ with $\delta_{i j}=1$ for $i=j, \delta_{i j}=0$ otherwise)

$$
\begin{aligned}
& A_{i k}:=E\left(-\frac{\partial^{2} \log (L)}{\partial x_{i} \partial x_{k}}\right)=\frac{\alpha n_{i+}}{x_{i}^{2}} \delta_{i k}, \quad 2 \leq i, k \leq m, \\
& B_{i j}:=E\left(-\frac{\partial^{2} \log (L)}{\partial x_{i} \partial y_{j}}\right)=\frac{\alpha n_{i j}}{x_{i} y_{j}}, \quad 2 \leq i \leq m, 1 \leq j \leq n, \\
& C_{l j}:=E\left(-\frac{\partial^{2} \log (L)}{\partial y_{l} \partial y_{j}}\right)=\frac{\alpha n_{+j}}{y_{j}^{2}} \delta_{l j}, \quad 1 \leq l, j \leq n,
\end{aligned}
$$

(where $n_{+j}$ includes $\left.n_{1 j}\right)$. With the matrices $A=\left(A_{i k}\right), B=\left(B_{i j}\right), C=\left(C_{l j}\right)$ the information matrix $I$ can be represented as partitioned matrix

$$
I=\left(\begin{array}{ll}
A & B \\
B^{t} & C
\end{array}\right)
$$

where $A$ and $C$ are diagnoal matrices.

Unfortunately, an explicit formula for the inverse matrix $I^{-1}$ is not available. One therefore must apply a numerical inversion method. But the dimension of the inversion problem can be reduced with the help of the following result for the inverse of a partitioned matrix (which can be verified by calculating $I^{-1} I$ and $\left.I I^{-1}\right)$ :

$$
\begin{aligned}
I^{-1} & =\left(\begin{array}{cc}
D^{-1} & -D^{-1} B C^{-1} \\
-C^{-1} B^{t} D^{-1} & C^{-1}+C^{-1} B^{t} D^{-1} B C^{-1}
\end{array}\right) \\
& =\left(\begin{array}{cc}
A^{-1}+A^{-1} B F^{-1} B^{t} A^{-1} & -A^{-1} B F^{-1} \\
-F^{-1} B^{t} A^{-1} & F^{-1}
\end{array}\right)
\end{aligned}
$$

with

$$
\begin{aligned}
& D=A-B C^{-1} B^{t}, \\
& F=C-B^{t} A^{-1} B .
\end{aligned}
$$

A straightforward calculation yields for the elements of $D$ and $F$

$$
\begin{aligned}
& D_{i k}=\alpha\left(\delta_{i k} n_{i+}-p_{i k}\right) /\left(x_{i} x_{k}\right), \quad 2 \leq i, k \leq m, \\
& F_{l j}=\alpha\left(\delta_{l j} n_{+j}-q_{l j}\right) /\left(y_{l} y_{j}\right), \quad 1 \leq l, j \leq n,
\end{aligned}
$$


with

$$
p_{i k}=\sum_{j=1}^{n} \frac{n_{i j} n_{k j}}{n_{+j}}, \quad q_{l j}=\sum_{i=2}^{m} \frac{n_{i l} n_{i j}}{n_{i+}} .
$$

Therefore, only the smaller matrices $D$ and $F$ must be inverted in order to obtain $I^{-1}$ and also $\hat{I}^{-1}$.

\section{ACKNOWLEDGEMENT}

I am very indebted to Peter Albrecht. I had an intensitve exchange of letters with him about this 1983 paper, which was very helpful to me. In addition, he drew my attention to some weak points in an earlier version of this paper. I would also like to thank PETER TER BERG for his advice on the digamma function and Alois Gisler for encouraging me to go ahead with this paper.

\section{REFERENCES}

Albrecht, P. (1983) Parametric Multiple Regression Risk Models. Insurance: Mathematics \& Economics, 49-66, 69-73 and 113-117.

Bailey, R.A. (1963) Insurance Rates with Minimum Bias. Proceedings of the Casualty Actuarial Society, 4-11.

BaIley, R. A. and Simon, L. J. (1960) Two Studies in Automobile Insurance Rate Making. $A S T I N$ Bulletin 1, 192-217.

Chang, L. and Fairley, W. B. (1979) Pricing Automobile Insurance under Multivariate Classification of Risks: Additive versus Multiplicative. Journal of Risk and Insurance, 75-98.

DE VYLDER, F. (1978) Estimation of IBNR Claims by Least Squares. Mitteilungen der Vereinigung Schweizerischer Versicherungsmathematiker, 249-254.

JEE, B. (1989) A Comparative Analysis of Alternative Pure Premium Models in the Automobile Risk Classification System. Journal of Risk and Insurance, 434 459.

JUNG, J. (1968) On Automobile Insurance Ratemaking. ASTIN Bulletin 5, 41-48.

Kremer, E. (1982) IBNR-Claims and the Two-way Model of ANOVA. Scandinavian Actuarial Journal, 47-55.

KREMER, E. (1985) Einführung in die Versicherungsmathematik. Vandenhoek \& Ruprecht, Göttingen. MACK, Th. (1990) Improved Estimation of IBNR Claims by Credibility Theory. Insurance: Mathematics \& Economics, 51-57.

RICHTER, H. (1966) Wahrscheinlichkeitstheorie. Springer, Heidelberg.

SANT, D. T. (1980) Estimating Expected Losses in Auto Insurance. Journal of Risk and Insurance, 133-151.

TAYLOR, G. C. (1986) Claims Reserving in Non-life Insurance. North Holland, Amsterdam. TER BERG, P. (1980) On the loglinear Poisson and Gamma model. ASTIN Bulletin 11, 3540. VAN EEghen, J. (1981) Loss Reserving Methods. Nationale-Nederlanden N.V., Rotterdam. van Eeghen, J., Greup, E. K. and Nijssen, J.A. (1983) Rate Making. Nationale-Nederlanden N.V., Rotterdam.

van Eeghen, J., Nijssen, J.A. and Ruygt, F. A.M. (1982) Interdependence of Risk Factors: Application of Some Models. New Motor Rating Structure in the Netherlands, 105-119. ASTINgroep, Nederland.

ZeHnwIRTH, B. (1989) The Chain Ladder Technique - A Stochastic Model. Claims Reserving Manual Vol. 2, 2-9. Institute of Actuaries, London.

Dr. Thomas Mack

Münchener Rückversicherungs-Gesellschaft, Königinstr. 107, D-8000 München 40, FRG. 